\title{
Guidelines for reporting mortality and morbidity after cardiac valve interventions
}

\author{
Cary W. Akins, MD, ${ }^{a}$ D. Craig Miller, MD, ${ }^{a}$ Marko I. Turina, MD, ${ }^{c}$ Nicholas T. Kouchoukos, MD, ${ }^{b}$
}

Eugene H. Blackstone, MD, a Gary L. Grunkemeier, PhD, ${ }^{b}$ Johanna J. M. Takkenberg, MD, PhD, ${ }^{c}$ Tirone E. David, MD, ${ }^{a}$ Eric G. Butchart, MD, ${ }^{c}$ David H. Adams, MD, ${ }^{b}$ David M. Shahian, MD, Siegfried Hagl, MD, ${ }^{c}$ John E. Mayer, MD, ${ }^{b}$ and Bruce W. Lytle, MDa

From The American Association for Thoracic Surgery, ${ }^{\text {a }}$ The Society of Thoracic Surgeons, ${ }^{\text {b }}$ and the European Association for Cardio-Thoracic Surgery. ${ }^{\mathrm{C}}$

This article is being published concurrently in The Annals of Thoracic Surgery and the European Journal of Cardio-Thoracic Surgery.

Received for publication Dec 11, 2007; accepted for publication Dec 11, 2007.

Address for reprints: Cary W. Akins, MD, Cardiac Surgery, Cox 648, Massachusetts General Hospital, 55 Fruit St, Boston, MA 02114 (E-mail: cakins@partners.org).

J Thorac Cardiovasc Surg 2008;135:732-8

0022-5223/\$34.00

Copyright $(92008$ by the American Association for Thoracic Surgery, the European Association for Cardio-Thoracic Surgery, and the Society of Thoracic Surgeons

doi:10.1016/j.jtcvs.2007.12.002
$\mathrm{S}$ ince the initial publication of "Guidelines for Reporting Morbidity and Mortality After Cardiac Valvular Operations" in 1988, ${ }^{1}$ followed by a revised version in $1996,{ }^{2}$ valvular heart surgery has evolved to include an enhanced understanding of patient- and disease-related factors affecting outcomes, increased numbers of valve repairs, more operations performed for patients with minimal symptoms, new prostheses, novel repair methods, and the emergence of percutaneous interventional (catheter-based) valve repair and replacement. To adapt to this changing environment, the Councils of the American Association for Thoracic Surgery, The Society of Thoracic Surgeons, and The European Association for Cardio-Thoracic Surgery have directed an Ad Hoc Liaison Committee for Standardizing Definitions of Prosthetic Heart Valve Morbidity to review current clinical practice to update and clarify these reporting guidelines. The guidelines are intended to cover treatment of all four cardiac valves in both adult and pediatric patients. Further, these guidelines apply uniformly, irrespective of whether the therapy was carried out as a conventional open operation, as a minimally invasive (video-assisted or robotic) surgical procedure, or with percutaneous interventional catheter techniques.

\section{Purpose}

These reporting guidelines are intended to facilitate analysis and reporting of clinical results of various therapeutic approaches to diseased heart valves such that meaningful comparisons can be made and inferences drawn from investigations of medical, surgi$\mathrm{cal}$, and percutaneous interventional treatment of patients with valvular heart disease.

\section{Early Mortality}

Early mortality is to be reported as all-cause mortality at 30,60, or 90 days and depicted by actuarial estimates (with number remaining at risk and confidence intervals [CIs]) or as simple percentages, regardless of the patient's location, be it home or in a health care facility.

\section{Definitions of Morbidity Structural Valve Deterioration}

Structural valve deterioration includes dysfunction or deterioration involving the operated valve (exclusive of infection or thrombosis), as determined by reoperation, autopsy, or clinical investigation. Clinical investigation should include periodic echocardiographic surveillance. Substantially increased regurgitation or stenosis of the 


\section{Abbreviation and Acronym}

$\mathrm{CI}=$ confidence interval

operated valve over time should be reported with quantitative or semiquantitative methods. The term structural valve deterioration refers to changes intrinsic to the valve, such as wear, fracture, poppet escape, calcification, leaflet tear, stent creep, and suture line disruption of components of a prosthetic valve; it also refers to new chordal rupture, leaflet disruption, or leaflet retraction of a repaired valve.

\section{Nonstructural Dysfunction}

Nonstructural dysfunction is any abnormality not intrinsic to the valve itself that results in stenosis or regurgitation of the operated valve or hemolysis. The term nonstructural dysfunction refers to problems (exclusive of thrombosis and infection) that do not directly involve valve components yet result in dysfunction of an operated valve, as diagnosed by reoperation, autopsy, or clinical investigation. Examples of nonstructural dysfunction include the following: entrapment by pannus, tissue, or suture; paravalvular leak; inappropriate sizing or positioning; residual leak or obstruction after valve implantation or repair; and clinically important intravascular hemolytic anemia. In addition, nonstructural dysfunction includes development of aortic or pulmonic regurgitation as a result of technical errors, dilatation of the sinotubular junction, or dilatation of the valve annulus after either valve replacement with stentless prostheses (eg, pulmonary autograft, aortic allograft, and xenograft valves) or aortic valve-sparing operations if the cusps are seen to be normal at reoperation, autopsy, or clinical investigation. For percutaneous and transapical approaches to aortic valve replacement or conventional open aortic valve replacement, new onset of coronary ischemia from coronary ostial obstruction or paravalvular aortic regurgitation is considered nonstructural dysfunction. More than mild recurrent or residual mitral or tricuspid regurgitation after surgical or percutaneous interventional valve procedures (coronary sinus interventions, direct reparative methods, or other methods aimed at achieving ventricular remodeling) is nonstructural dysfunction, unless there is disruption of the valve components themselves, which would then be structural deterioration.

Sudden or progressive dysfunction or deterioration of the operated valve may be structural, nonstructural, or both, as determined by reoperation, autopsy, or clinical investigation.

\section{Valve Thrombosis}

Valve thrombosis is any thrombus not caused by infection attached to or near an operated valve that occludes part of the blood flow path, interferes with valve function, or is sufficiently large to warrant treatment. Valve thrombus found at autopsy in a patient whose cause of death was not valve related or found at operation for an unrelated indication should also be counted as valve thrombosis.

\section{Embolism}

Embolism is any embolic event that occurs in the absence of infection after the immediate perioperative period. Embolism may be manifested by a neurologic event or a noncerebral embolic event.

A neurologic event includes any central, new neurologic deficit, whether temporary or permanent and whether focal or global, that occurs after the patient emerges from anesthesia.

Stroke is a prolonged ( $>72$ hours) or permanent neurologic deficit that is usually associated with abnormal results of magnetic resonance imaging or computed tomographic scans. Patients with minimal, atypical, or protean symptoms that lead to radiographic imaging demonstrating an acute ischemic event are considered to have sustained a stroke.

Transient ischemic attack is characterized by fully reversible symptoms of short duration. If radiographic imaging demonstrates an acute central neurologic lesion ("cerebral infarction with transient symptoms"), however, such patients are reclassified as having sustained a stroke.

Multiple or repeated transient events occurring during a short period (a burst or cluster) should be recorded as one event for calculation of event rates, but documented as a cluster. Rate calculations should be provided not only for all embolic events but also separately for strokes, transient ischemic attacks, and clusters.

Postoperative neurologic symptoms that mimic those of a preoperatively documented neurologic event and that are confirmed radiographically to be consistent with the former event are not counted as a new neurologic event. Central neurologic events that are clearly related to aortic, internal carotid artery, or vertebral artery disease, such as acute thrombotic occlusion, atheroembolism, or spontaneous arterial dissection, are also not counted. Psychomotor deficits found by specialized testing are not considered neurologic events related to operated valves. Patients who do not awaken or who awaken after operation with a new stroke are not considered to have sustained valve-related neurologic events.

A noncerebral embolic event is an embolus documented operatively, at autopsy, or clinically that produces signs or symptoms attributable to complete or partial obstruction of a peripheral artery. Intraoperative myocardial infarctions are not counted. Postoperative myocardial infarction is also not counted unless the infarction is caused by a coronary embolus (as detected by operation, autopsy, or clinical imaging). Emboli consisting of nonthrombotic material (eg, atherosclerosis, myxoma) are not counted.

\section{Bleeding Event}

A bleeding event is any episode of major internal or external bleeding that causes death, hospitalization, or permanent 
injury (eg, vision loss) or necessitates transfusion. Major bleeding unexpectedly associated with minor trauma should be reported as a bleeding event, but bleeding associated with major trauma or a major operation should not. Bleeding events are reported for all patients regardless of whether they are taking anticoagulants or antiplatelet drugs. Although total bleeding events must be reported, bleeding events can also be reported separately for those who are taking anticoagulants or antiplatelet agents and those who are not.

\section{Antithrombotic Management}

The method of initiating antithrombotic treatment during hospitalization should be specified (eg, intravenous unfractionated heparin, subcutaneous low-molecular-weight heparin, antiplatelet agent). If oral anticoagulant therapy is instituted, the following information should be specified: (1) specific drug used (eg, warfarin sodium, acenocoumarol, phenprocoumon), (2) target international normalized ratio for each valve position, (3) average achieved international normalized ratio, (4) method of anticoagulation control (eg, physician or nurse directed, patient home self-management), and (5) duration of treatment for patients with bioprostheses. If antiplatelet drugs are used, they should be specified.

If the patient has a valve thrombosis, embolism, or bleeding event, the international normalized ratio associated with that event should be reported, together with any antiplatelet therapy.

\section{Composite Thrombosis, Embolism, and Bleeding}

The composite end point of thrombosis, embolism, and bleeding includes occurrence of all events meeting the previously stated definitions of valve thrombosis, embolism, and bleeding event. Because thrombogenicity and intensity of anticoagulation may be manifested by several separate complications, this composite end point represents a more accurate overall assessment of the total hazard of thrombogenicity and anticoagulation. ${ }^{3}$

\section{Operated Valve Endocarditis}

Operated valve endocarditis is any infection involving a valve on which an operation has been performed. The diagnosis of operated valvular endocarditis is based on one of the following criteria: (1) reoperation with evidence of abscess, paravalvular leak, pus, or vegetation confirmed as secondary to infection by histologic or bacteriologic studies; (2) autopsy findings of abscess, pus, or vegetation involving a repaired or replaced valve; or (3) in the absence of reoperation or autopsy, meeting of the Duke Criteria for endocarditis. ${ }^{4}$ Positive blood cultures are not required for the diagnosis of operated valve endocarditis. Culture-negative endocarditis should refer only to negative blood culture results and not just the absence of any proof of infection. Morbidities associated with active infection, such as valve thrombosis, thrombotic embolus, bleeding event, or paravalvular leak, are included under this category, but not counted in other categories of morbidity.

\section{Reintervention}

Reintervention is any surgical or percutaneous interventional catheter procedure that repairs, otherwise alters or adjusts, or replaces a previously implanted prosthesis or repaired valve. In addition to surgical reoperations, enzymatic, balloon dilatation, interventional manipulation, repositioning, or retrieval, and other catheter-based interventions for valverelated complications are also considered reinterventions. Indications for reintervention must be reported. Open surgical and percutaneous catheter reinterventions should be listed separately.

\section{Valve-Related Mortality}

Valve-related mortality is any death caused by structural valve deterioration, nonstructural dysfunction, valve thrombosis, embolism, bleeding event, or operated valve endocarditis; death related to reintervention on the operated valve; or sudden, unexplained death. Deaths caused by heart failure in patients with advanced myocardial disease and satisfactorily functioning cardiac valves are not counted. Specific causes of valve-related deaths should be reported.

\section{Sudden, Unexplained Death}

A sudden, unexplained death is one in which the cause of death has not been determined by clinical investigation or autopsy findings and the relationship to the operated valve is undefined. These deaths should be reported as a separate category, but also included in valve-related mortality.

\section{Cardiac Death}

Cardiac death includes all deaths resulting from cardiac causes. This category includes valve-related deaths, sudden unexplained deaths, and deaths from non-valve-related cardiac causes (eg, from heart failure, acute myocardial infarction, or documented arrhythmias).

\section{All-Cause Mortality}

All-cause mortality includes all deaths from any cause after a valve intervention. Survival should be referenced to an age- and sex-matched sample from the representative general population being investigated whenever possible.

\section{Permanent Valve-Related Impairment}

Permanent valve-related impairment is any permanent neurologic or other functional deficit caused by structural valve deterioration, nonstructural dysfunction, valve thrombosis, embolism, bleeding event, operated valve endocarditis, or reintervention. 


\section{Major Adverse Valve-Related Event}

Major adverse valve-related events include the following: (1) valve-related mortality, (2) all valve-related morbidity, and (3) need for new permanent pacemaker or defibrillator within 14 days after the valve intervention.

\section{Data Collection}

Data collection and reporting for all treated valves should include location (aortic, mitral, tricuspid, pulmonary, multiple), treatment method (repair, replacement, percutaneous catheter intervention), repair methods if valve preserved (including type of annuloplasty ring, suture annuloplasty, or coronary sinus cerclage), and, for valve replacement, prosthesis type (mechanical prosthesis, stented bovine pericardial or porcine bioprosthesis, stentless xenograft bioprosthesis, aortic or pulmonary allograft, pulmonary autograft). For prostheses, including annuloplasty rings, manufacturer and model should be reported. For allografts, method of preservation should be given. Manufacturer label size should be stated for each valve location, type, and model; in addition, calibrated annulus size (or maximal dilatation balloon diameter during preliminary balloon valvuloplasty and during valve deployment in cases of percutaneous aortic valve replacement) before valve implantation should also be reported. Not only should the number of treated valves be listed, so too should the number of patients who received them.

\section{Additional Pertinent Material}

In addition, the report should specify the following:

1. The patient population from which the study cohort was selected, preferably according to CONSORT (Consolidated Standards of Reporting Trials) recommendations (see http://www.consort-statement.org). Inclusive dates of operation and whether the series was consecutive should be stated. Criteria used to select patients should be defined and listed. If a subset of the sample population is reported, the total number of patients who underwent valve intervention during the inclusive dates of the clinical investigation should be reported.

2. The method used for follow-up. This includes type of follow-up, which may be active (direct contact with patients or their families by examination, telephone, letter, or questionnaire) or passive (use of administrative or government data not involving direct patient contact). Mode of follow-up should be included, whether prospective anniversary contact (although periodic follow-up may be at intervals shorter or longer than 1 year) or cross-sectional, whereby an entire group of patients is followed up more or less at the same calendar time despite their index procedures having occurred at widely disparate times.
3. Percentages of patient-level responses from each method should be given. In case of an anniversarytype follow-up, frequency of follow-up inquiry should be provided.

4. Total follow-up time (patient-years), mean (and SD) or median (and quartiles) if the distribution of data is skewed, and maximum years of follow-up should be given. If the study involves multiple valve positions, treatment methods, repair techniques, and prosthesis types, total follow-up time for each should be reported separately.

5. The time period (closing interval) required to complete current follow-up should be given if the common closing date method is not used. The closing interval, in which the current status of all patients is determined, should be as short as possible. Alternatively, the status of all patients at their exact anniversary dates, or as of the receipt of the first response to a cross-sectional inquiry, may be used as a common closing date.

6. Completeness of follow-up can be calculated as the ratio of total observed person-time to potential person-time of follow-up to the closing date of the study. ${ }^{5}$ Although follow-up to death (or explant in a valve-oriented analysis) is $100 \%$ complete, because of deaths, observable patient-years will be less than potential patient-years. A modification can be made of Clark and colleagues' $\mathrm{C}$ statistic to account for this, which will yield a somewhat higher percentage. ${ }^{6}$ To improve statistical validity, every effort should be made to achieve complete current follow-up for more than $90 \%$ of patients.

7. Percentage of autopsies and documented modes of death should be reported.

\section{Data Analysis and Reporting}

The method of reporting data should facilitate comparison between reports and support the conclusions, inferences, and predictions made. Methods chosen to analyze the collected data depend on the purpose of the report and availability of analytic techniques. Methods used to collect and analyze data should be summarized in the Methods section, with references included or defined in an appendix.

\section{Percentages (Not Time Related)}

Some morbid events occurring within a short time frame may be reported as simple percentages, that is, the number of events divided by the number of patients (eg, 30-, 60-, or 90-day mortality), as long as the status of all patients is known. Percentages should be presented with $\mathrm{CIs}^{7}$ and may be compared by Pearson's $\chi^{2}$ test or Fisher's exact test. ${ }^{8}$ Logistic regression analysis ${ }^{9}$ is available for evaluating the simultaneous influence of several risk factors on a dichotomous outcome variable (percentage) and is often used to establish 
a risk model, that is, a mathematical formula incorporating such factors.

\section{Time-Related Events}

Valve-related events should be reported in a time-related manner, with time of treatment designated as time zero. Kaplan-Meier ${ }^{10}$ or other life table techniques ${ }^{11}$ provide actuarial estimates of morbid events, and these should be reported with 1 or 2 SEs of the estimate (equivalent to $68 \%$ or $95 \%$ CIs). Number of patients remaining at risk should be indicated at appropriate intervals, and curves should use dashed lines beyond time frames containing few patients, such as $10 \%$ of the initial cohort in a typical-size (hundreds, not thousands, of patients) study. Although comparisons between subsets of patients can be made, actuarial methods are not predictive beyond the time of the last actuarial estimate and cannot be adapted to multivariable analysis. These methods are called nonparametric or distribution free because they do not assume a particular statistical distribution or model.

\section{Risk Factors}

The Cox proportional hazards model ${ }^{12}$ produces a timedependent analysis of valve-related events and provides a multivariable regression method to discriminate risk factors associated with specific valve-related morbid events during specific intervals. The Cox method is a semiparametric (model partially specified) approach that makes no assumption about the shape of the underlying hazard function, but identifies risk factors and estimates multipliers of the baseline hazard. These multipliers are the relative risks (called hazard ratios) associated with the risk factors. Several methods are available for assessing the assumption of proportional hazards. ${ }^{13}$ When such methods reject the hypothesis of proportional hazards, one can be reasonably sure that the method is inappropriate and alternatives to it are needed; if the hypothesis is not rejected, one unfortunately has not learned much, because these methods are sensitive to number of events and tend to be conservative.

Results of a multivariable analysis should be accompanied by a list of the variables considered and a tabular presentation of the numeric results. When modeling event risk (by either logistic or Cox regression), the amount of information available is based on number of events, not number of patients or patient-years. Thus, it is important that a sufficient number of events occur to enable accurate estimates. A "rule of 10 " events as the minimum per risk factor considered in the model has been advocated for both logistic regression ${ }^{14}$ and Cox regression, ${ }^{15}$ although this minimum could be lowered a bit. ${ }^{16}$ In cases of few events per risk factor, resampling techniques can be used to test model validity.

\section{Temporal Pattern of Risk}

A fully parametric method (model completely specified) of calculating a hazard function of valve-related morbid events defines the instantaneous risk of an event at any time after treatment. ${ }^{17-20}$ Such methods permit univariable and multivariable analysis (including those specific to various time frames, such as early vs long-term risk), provide predictive information beyond the time of the last event, indicate whether the risk is constant, and provide CIs. For example, the hazard function for structural valve deterioration for bioprostheses is not constant across time, but increases with time since insertion; a Weibull function that accommodates an increasing hazard with time should be considered. ${ }^{21,22}$

\section{Linearized rates}

If the risk of an event is constant over time, there is a simple method to calculate that rate. The linearized rate is calculated as total number of observed events divided by total patientyears of follow-up. It is often expressed as events per 100 patient-years (percent per year). These rates should be considered only approximate unless the hazard function for the complication under study is constant during the entire interval considered, which is often not true for complications after a cardiac valve procedure. Linearized rates should be reported with CIs, which can be based on the Poisson distribution $^{23}$ or on likelihood ratio methods for comparing the means of exponential distributions. ${ }^{11,23}$ Linearized rates can be compared with the likelihood ratio test, ${ }^{19,20,24}$ a test that is based on the $F$ statistic, ${ }^{12,20}$ or within appropriate multivariable models.

\section{Repeated events}

Some valve-related events, such as thromboembolism and bleeding, can occur repeatedly. Although estimating freedom from any such event is meaningful, even more important is enumerating all such events. Some of the previously mentioned methods have been devised or extended to consider repeated events in the same patient. ${ }^{17,25,26}$ A simple and widely used approach uses linearized rates, as described previously, to estimate the incidence of multiple events. These rates should be considered only approximate unless the risk of recurrent events is the same as for initial events (which is often not the case). If it is not, a simple approach is to restart time zero at each event occurrence.

\section{Valve Outcome Versus Patient Outcome}

Time-related events that estimate valve performance are measured from time of treatment until time of patient death, valve explantation, or censoring; however, patients are interested in learning what events they may encounter during the remainder of their lives. Thus, patient outcome should be measured starting at the time of treatment until death (or censoring). Because patient death competes with event occurrence, it is important to make a clear distinction between valve performance and patient outcome. Because the Kaplan-Meier method assumes patient immortality when estimating cumulative freedom from events, it overestimates 
the actual probability of event occurrence for the patient (or a particular patient population). To translate valve outcome (valve performance) to patient outcome (risk of an adverse event), the cumulative incidence method is recommended. For assessment of valve prosthesis performance or durability of repair, which focuses on the valve rather than the patient as the unit of measure, Kaplan-Meier and related actuarial methods are appropriate, rather than cumulative incidence methods. ${ }^{27}$ The Kaplan-Meier method is usually used to estimate occurrence of valve-related events. This method is not perfect, because it assumes independence between death and the event of interest, which in most instances is not true. Inverse probability weighting may correct for this to provide a better estimate of true valve performance. ${ }^{28}$

\section{Longitudinal Outcomes}

Time-related events after valve replacement are assumed to occur at an instant in time; however, many outcomes of importance are conditions or processes that evolve with time, such as return of regurgitation after valve repair, change in New York Heart Association functional class (graded or ordinal outcomes), regression of ventricular mass (continuous outcome), and use of warfarin sodium (binary outcome). ${ }^{29}$ Values for these outcomes are captured at discrete instances in time ("snapshots"), which may be taken repeatedly at prospectively specified follow-up intervals, cross-sectionally, or opportunistically.

Snapshots are subject to many biases. If a condition changes rapidly but snapshots are taken infrequently, aliasing is introduced. If opportunistic follow-up occurs only when symptoms recur, the prevalence of undesirable change may be overestimated. Change is also related to precision of measurements; for example, degree of mitral regurgitation depends on systolic blood pressure and quality of the echocardiogram. Of course, the entire series of assessments is truncated by death or by removal of the valve of interest.

The challenge in analyzing longitudinal data is estimating the average temporal pattern of outcome and its variability in the group of patients. This average must account for sampling challenges, censoring (truncation) by death, unequal number of repeated measurements of the outcome per patient, variability in time among repeated measurements (such as serial echocardiographic assessment at different intervals after treatment), and the fact that sequential measurements obtained for a given patient will be more correlated with themselves than will measurements between individuals. Thus, these kinds of data, methods of longitudinal data analysis have developed rapidly during the last two decades. ${ }^{30}$ These methods include mixed models, random and fixed effects models, generalized estimating equation approach, and hierarchic models (such as currently used for The Society of Thoracic Surgeons National Database risk assessment). ${ }^{31-33}$ Longitudinal data analysis of a series of assessments is superior to analyzing only condition at last follow-up. This methodology is also superior to dichot- omizing outcomes and analyzing them with actuarial methods as if they were events, such as freedom from grade $3+$ mitral regurgitation after repair.

We acknowledge the invaluable assistance given by Cindy VerColen of The American Association for Thoracic Surgery for the organization of this review and development of this document.

\section{References}

1. Edmunds LH Jr, Cohn LH, Weisel RD. Guidelines for reporting morbidity and mortality after cardiac valvular operations. J Thorac Cardiovasc Surg. 1988;96:351-3.

2. Edmunds LH Jr, Clark RE, Cohn LH, Grunkemeier GL, Miller DC, Weisel RD. Guidelines for reporting morbidity and mortality after cardiac valvular operations. Ad Hoc Liaison Committee for Standardizing Definitions of Prosthetic Heart Valve Morbidity of The American Association for Thoracic Surgery and The Society of Thoracic Surgeons. J Thorac Cardiovasc Surg. 1996;112:708-11.

3. Akins CW. Results with mechanical cardiac valvular prostheses. Ann Thorac Surg. 1995;60:1836-44.

4. Li JS, Sexton DJ, Mick N, Nettles R, Fowler VG Jr, Ryan T, et al. Proposed modifications to the Duke criteria for the diagnosis of infectious endocarditis. Clin Infect Dis. 2000;30:633-8.

5. Clark TG, Altman DG, De Stavola BL. Quantification of the completeness of follow-up. Lancet. 2002;359:1309-10.

6. Wu YX, Takkenberg JJ, Grunkemeier GL. Measuring follow-up completeness. Ann Thorac Surg. 2008. In press.

7. Vollset SE. Confidence intervals for a binomial proportion. Stat Med. 1993; 12:809-24.

8. Fleiss JL, Levin B, Paik MC. Statistical methods for rates and proportions. 3rd ed. New York: Wiley Interscience; 2003.

9. Hosmer DW, Lemeshow S. Applied logistic regression. 2nd ed. New York: Wiley Interscience; 2000.

10. Kaplan EL, Meier P. Nonparametric estimation from incomplete observations. J Am Stat Assoc. 1958;53:457-81.

11. Cutler SJ, Ederer F. Maximum utilization of the life table method in analyzing survival. J Chron Dis. 1958;8:699-712.

12. Cox DR, Oakes D. Analysis of survival data. London: Chapman and Hall; 1984.

13. Hess KR. Graphical methods for assessing violations of the proportional hazards assumption in Cox regression. Stat Med. 1995;14:1707-23.

14. Peduzzi P, Concato J, Kemper E, Holford TR, Feinstein AR. A simulation study of the number of events per variable in logistic regression analysis. J Clin Epidemiol. 1996;49:1373-9.

15. Peduzzi P, Concato J, Feinstein AR, Holford TR. Importance of events per independent variable in proportional hazards regression analysis. II. Accuracy and precision of regression estimates. J Clin Epidemiol. 1995; 48:1503-10.

16. Vittinghoff $\mathrm{E}, \mathrm{McCulloch} \mathrm{CE}$. Relaxing the rule of ten events per variable in logistic and Cox regression. Am J Epidemiol. 2007;165:710-8.

17. Blackstone EH, Naftel DC, Turner ME Jr. The decomposition of timevarying hazard into phases, each incorporating a separate stream of concomitant information. J Am Stat Assoc. 1986;81:615-24.

18. Cohen A, Whitten BJ. Parameter estimation in reliability and life span models. New York: Marcel Dekker; 1988

19. Lawless JF. Statistical models and methods for lifetime data. 2nd ed. New York: John Wiley; 2003.

20. Gross AJ, Clark VA. Survival distributions: reliability applications in the biomedical sciences. New York: John Wiley; 1975.

21. Banbury MK, Cosgrove DM 3rd, White JA, Blackstone EH, Frater RW, Okies JE. Age and valve size effect on the long-term durability of the Carpentier-Edwards aortic pericardial bioprosthesis. Ann Thorac Surg. 2001;72:753-7.

22. Grunkemeier G, Li HH, Naftel DC, Starr A, Rahimtoola SH. Long-term performance of heart valve prostheses. Curr Probl Cardiol. 2000;25: 73-154.

23. Sahai H, Khurshid A. Confidence intervals for the mean of a Poisson distribution: a review. Biom J. 1993;35:857-67. 
24. Lee ET, Wang JW. Statistical methods for survival data analysis. New York: John Wiley; 2003.

25. Nelson W. Graphical analysis of system repair data. J Qual Technol. 1988;20:24-35.

26. Nelson W. Hazard plotting for incomplete failure data. J Qual Technol. 1969; 1:27-52.

27. Bodnar E, Blackstone EH. Editorial: an 'actual' problem: another issue of apples and oranges. J Heart Valve Dis. 2005;14:706-8.

28. Grunkemeier GL, Jin R, Eijkemans MJ, Takkenberg JJ. Actual and actuarial probabilities of competing risks: apples and lemons. Ann Thorac Surg. 2007;83:1586-92.
29. Banbury MK, Cosgrove DM 3rd, Thomas JD, Blackstone EH, Rajeswaran J, Okies JE, et al. Hemodynamic stability during 17 years of the Carpentier-Edwards aortic pericardial bioprosthesis. Ann Thorac Surg. 2002;73:1460-5.

30. Diggle PJ, Heagerty PJ, Liang KY, Zeger SL. Analysis of longitudinal data. 2nd Ed. New York: Oxford University Press; 2002.

31. Laird NM, Ware JH. Random-effects models for longitudinal data. Biometrics. 1982;38:963-74.

32. Morris CN. Parametric empirical Bayes inference: theory and applications. J Am Stat Assoc. 1983;78:47-55.

33. Goldstein H. Multilevel statistical models, 3rd ed. London: Edward Arnold; 2003 